\title{
EMITTANCE GROWTH CAUSED BY BUNCHED BEAM WITH NONUNIFORM DISTRIBUTIONS IN BOTH LONGITUDINAL AND TRANSVERSE DIRECTIONS IN LINAC*
}

\author{
Zhibin Huang, Yinbao Chen, Shinian Fu \\ ( China Institute of Atomic Energy, P. O. Box 275-17, Beijing 102413, China )
}

\begin{abstract}
The nonlinear space charge effect of bunched beam in linac is one of the important factors that induces the emittance growth due to the conversion of the field energy to kinetic energy. In this paper, using a cylinder model of space charge in linac, we derived the internal energy for a bunched beam with some nonuniform space charge distributions, such as Gaussian, waterbag and parabolic distributions in both longitudinal and transverse directions. And the emittance growth caused by these nonuniformities is worked out.
\end{abstract}

\section{INTRODUCTION}

The nonlinear space charge effect in a charged beam is one of the basic factors determining the beam dynamics in many intense beam facilities, such as Free Electron Laser (FEL), Inertial Confined Fusion (ICF), Heavy Ion Fusion (HIF) and some microwave devices. Theoretical and experimental studies on the intense beam have already regarded nonuniform charge distribution as a major cause of emittance growth, a nonuniform beam have higher field energy than the equivalent beam, and the particle distribution will become uniform. The difference in potential energy is converted to kinetic energy as the distribution tends to more homogeneity. A general relationship between the possible emittance growth and excess energy is given in Ref.[1]. Formulae for calculating the nonlinear self-field energy of a space charge bunched beam with nonuniform distribution in transverse direction in linac are given in Ref.[2], and hence emittance growth caused by nonuniformities is discussed, but in the above discussion uniform distribution in longitudinal direction is assumed. Therefore, it is necessary to derive formulae for calculating the nonlinear field energy of a space charge bunched beam with the nonuniform distributions in both longitudinal and transverse directions and to calculate the possible emittance growth caused by the nonuniformities. Using a cylinder model of space charge in linac, we derived formulae for calculating the self-field energy of a space charge bunched beam with each of different longitudinal distributions such as waterbag and parabolic distributions combining with each of different transverse distributions such as Kapchinskij-Vladimirskij (K-V), waterbag (WB), parabolic (PA) and Gaussian (GA) distributions, and gave curves of the corresponding emittance growth.

\section{SELF-FIELD ENERGY FOR BUNCHED BEAM WITH LONGITUDINAL NONUNIFORM DISTRIBUTION}

A cylinder model of space charge is used here to present a space charge in linac. In the waveguide of a linac, assume the cylinder model to be symmetry and let the cylindrical space charge $\mathrm{q}$ be set in the central position, $\mathrm{b}$ and $\pm \mathrm{L} / 2$ are boundaries of the bunch in $\mathrm{r}$ and $\mathrm{z}$ axes respectively.

According to the cylindrical density distribution and the potential induced by some nonuniform distributions in Ref.[3], using the formula for calculating total stationary field energy in linear medium as

$$
W=\frac{1}{2} \int \rho \phi d \tau,
$$

where $\rho$ stands for the charge density, $\phi$, the potential and $\mathrm{d} \tau=\operatorname{rdrd} \theta \mathrm{d} z$. we can get the self-field energy corresponding to each of different longitudinal distributions combining with each of different transverse distributions.

\section{A. Longitudinal Waterbag Distribution}

For transverse K-V distribution we obtain the selffield energy as below:

$$
W_{k v, w b}=\frac{18 q^{2} a^{2}}{\pi \varepsilon_{0} L b^{2}} \sum_{l=1}^{\infty} \frac{J_{1}^{2}\left(k_{l} b\right)}{\left(k_{l} a\right)^{4} J_{1}^{2}\left(k_{l} a\right)} A_{w b},
$$

where the subscript in which before the comma presents transverse distribution and after the comma longitudinal

* The project is supported by National Natural Science Foundation of China (NSFC) and China Science Foundation of Nuclear Industry. 
distribution, $\mathrm{a}$ is the radius of the waveguide, $\mathrm{b}$ and $\mathrm{L}$ are the radius and length of the cylinder, respectively. $\mathrm{J}_{i}\left(\mathrm{k}_{l} \mathrm{x}\right)$ is the Bessel function and $\mathrm{k}_{l}$ satisfies the equation: $\mathrm{J}_{0}\left(\mathrm{k}_{l} \mathrm{a}\right)=0$. Let $\mathrm{C}=\mathrm{k}_{l} \mathrm{~L} / 2, A_{w b}$ stands for

$$
A_{w b}=\frac{2}{15}-\frac{1}{3 C^{2}}+\frac{1}{C^{3}}\left[1+\frac{1}{C}-\left(1+\frac{1}{C}\right)^{2} e^{-C} s h C\right],
$$

For transverse WB distribution we get the corresponding self-field energy as below:

$$
W_{w b, w b}=\frac{288 q^{2} a^{2}}{\pi \varepsilon_{0} L b^{4}} \sum_{l=1}^{\infty} \frac{J_{2}^{2}\left(k_{l} b\right)}{\left(k_{l} a\right)^{6} J_{1}^{2}\left(k_{l} a\right)} A_{w b} .
$$

For transverse PA distribution we have the corresponding self-field energy as below:

$$
W_{p a, w b}=\frac{10368 q^{2} a^{2}}{\pi \varepsilon_{0} L b^{6}} \sum_{l=1}^{\infty} \frac{J_{3}^{2}\left(k_{l} b\right)}{\left(k_{l} a\right)^{8} J_{1}^{2}\left(k_{l} a\right)} A_{w b} .
$$

For transverse GA distribution we obtain the corresponding self-field energy as below:

$$
W_{g a, w b}=\frac{9 q^{2}}{2 \pi \varepsilon_{0} L} \sum_{l=1}^{\infty} \frac{e^{-k_{l}^{2} \alpha^{2}}}{\left(k_{l} a\right)^{2} J_{1}^{2}\left(k_{l} a\right)} A_{w b} .
$$

\section{B. Longitudinal Parabolic Distribution}

For transverse K-V distribution we get the corresponding self-field energy as below:

$$
W_{k v, p a}=\frac{20 q^{2} a^{2}}{7 \pi \varepsilon_{0} L b^{2}} \sum_{l=1}^{\infty} \frac{J_{1}^{2}\left(k_{l} b\right)}{\left(k_{l} a\right)^{4} J_{1}^{2}\left(k_{l} a\right)} A_{p a} .
$$

where $A_{p a}$ stands for

$$
\begin{aligned}
A_{p a}= & 1-\frac{3}{C^{2}}+\frac{63}{2 C^{4}}+\frac{954}{2 C^{6}}+\frac{2835}{2 C^{7}}+\frac{2835}{2 C^{8}}- \\
& -\frac{315}{2 C^{5}}\left(1+\frac{3}{C}+\frac{3}{C^{2}}\right)^{2} e^{-C} \operatorname{sh} .
\end{aligned}
$$

For transverse WB distribution we have the corresponding self-field energy as below:

$$
W_{w b, p a}=\frac{320 q^{2} a^{4}}{7 \pi \varepsilon_{0} L b^{4}} \sum_{l=1}^{\infty} \frac{J_{2}^{2}\left(k_{l} b\right)}{\left(k_{l} a\right)^{6} J_{1}^{2}\left(k_{l} a\right)} A_{p a} .
$$

For transverse PA distribution we obtain the corresponding self-field energy as below:

$$
W_{p a, p a}=\frac{11520 q^{2} a^{6}}{7 \pi \varepsilon_{0} L b^{6}} \sum_{l=1}^{\infty} \frac{J_{3}^{2}\left(k_{l} b\right)}{\left(k_{l} a\right)^{8} J_{1}^{2}\left(k_{l} a\right)} A_{p a} .
$$

For transverse GA distribution we get the corresponding self-field energy as below:

$$
W_{g a, p a}=\frac{5 q^{2}}{7 \pi \varepsilon_{0} L} \sum_{l=1}^{\infty} \frac{e^{-k_{l}^{2} \alpha^{2}}}{\left(k_{l} a\right)^{2} J_{1}^{2}\left(k_{l} a\right)} A_{p a}
$$

As L/2 tends to infinity, above formulae for calculating the self-field energy retrograde into those formulae $^{[2]}$ with correspondence to each of above referred distributions combining with longitudinal uniform distribution.

\section{EMITTANCE GROWTH}

In uniform beam the cylindrical space charge holds the self-field energy as below ${ }^{[2]}$ :

$$
W_{k v, k v}=\frac{2 q^{2} a^{2}}{\pi \varepsilon_{0} L b^{2}} \sum_{l=1}^{\infty} \frac{J_{1}^{2}\left(k_{l} b\right)}{\left(k_{l} a\right)^{4} J_{1}^{2}\left(k_{l} a\right)} A_{k v},
$$

where $A_{k v}$ stands for ${ }^{[2]}$

$$
A_{k v}=1-\frac{1}{C} e^{-C} s h C
$$

Now let $\mathrm{W}_{0}$ be equal to $\mathrm{W}_{\mathrm{kv}, \mathrm{kv}}$, then an equation can be described as below:

$$
U_{n}=W_{n}-W_{0},
$$

where the subscript $\mathrm{n}$ may be (kv,wb), (wb,wb), (pa,wb), (ga,wb), (kv,pa), (wb,pa), (pa,ap), or (ga,pa), respectively. $\mathrm{U}_{\mathrm{n}}$ is the difference between the nonuniform distribution and the equivalent $\mathrm{k}-\mathrm{v}$ distribution in the self-field energy. The excess field energy is transformed into particle transverse kinetic energy as the distribution inclines to become more homogeneous, causing emittance growth. Provided that $\mathrm{U}_{\mathrm{n}} / \mathrm{W}_{0}$ would be expressed as the relative measurement of the excess field energy in nonuniform density distribution of a space charge bunched beam, using the formulae of the self-field energy, for some different distributions we can get $\mathrm{U}_{\mathrm{n}} / \mathrm{W}_{0}$.

The general formula for calculating the possible emittance growth caused by a space charge with nonuniform density distribution is given in Ref.[1]. The relationship between the possible emittance growth and $\mathrm{U}_{\mathrm{n}} / \mathrm{W}_{0}$ is described as below ${ }^{[1]}$

$$
\frac{\epsilon_{f}}{\epsilon_{i}}=\left[1+\frac{U_{n}}{2 W_{0}}\left(\frac{k_{0}^{2}}{k_{i}^{2}}-1\right)\right]^{\frac{1}{2}},
$$

where $\varepsilon_{\mathrm{i}}$ and $\varepsilon_{\mathrm{f}}$ are respectively assumed to be initial and final value of emittance, $\mathrm{k}_{0}$ represents the external focusing constant and $\mathrm{k}_{\mathrm{i}}$ the initial focusing constant. Substituting $\mathrm{U}_{\mathrm{n}} / \mathrm{W}_{0}$ into eq.(15) and assuming $\alpha / \mathrm{b}$ to be 0.3 in Gaussian 
distribution, we can obtain curves of the corresponding emittance growth $\varepsilon_{\mathrm{f}} / \varepsilon_{\mathrm{i}}$ versus the ratio b/a. As $\mathrm{k}_{\mathrm{i}} / \mathrm{k}_{0}$ equals 0.3 , figure 1 shows the possible emittance growth $\varepsilon_{\mathrm{f}} / \varepsilon_{\mathrm{i}}$ versus b/a for either waterbag or parabolic longitudinal distribution combining with each of different transverse distributions such as waterbag, parabolic and Gaussian distributions, figure 2 shows the possible emittance growth $\varepsilon_{\mathrm{f}} / \varepsilon_{\mathrm{i}}$ versus $\mathrm{L} / \mathrm{a}$ for $\mathrm{K}-\mathrm{V}$ transverse distribution combining with each of different longitudinal distributions such as waterbag and parabolic distributions. In additional, from eq.(15) we find that the possible emittance growth also varies with the ratio $\mathrm{k}_{\mathrm{i}} / \mathrm{k}_{0}$, As an example, figure 3 shows the possible emittance growth $\varepsilon_{\mathrm{f}} / \varepsilon_{\mathrm{i}}$ versus b/a for Gaussian transverse distribution combining with either waterbag or parabolic longitudinal distribution when $\mathrm{k}_{\mathrm{i}} / \mathrm{k}_{0}$ is equal to $0.1,0.2$ or 0.3 , respectively.

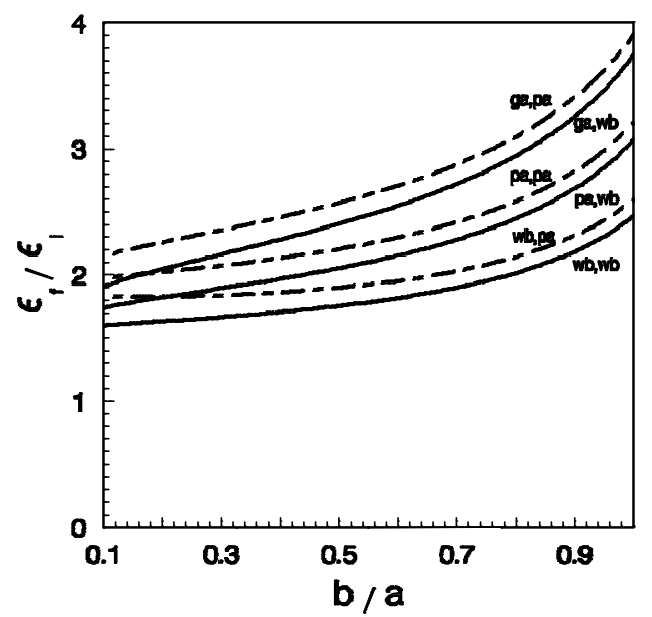

Figure 1: Emittance growth $\varepsilon_{\mathrm{f}} / \varepsilon_{\mathrm{i}}$ vs b/a for different longitudinal distributions combining with different transverse distributions.

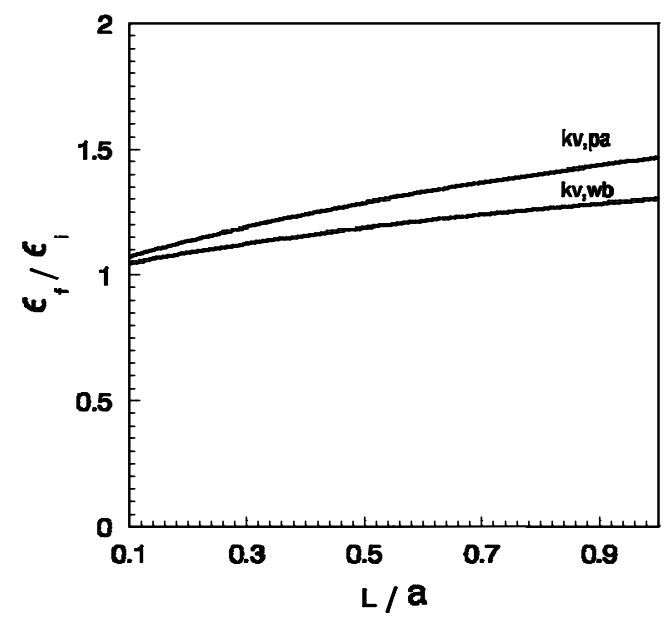

Figure 2: Emittance growth $\varepsilon_{\mathrm{f}} / \varepsilon_{\mathrm{i}}$ vs L/a for transverse K$\mathrm{V}$ distribution combining with different longitudinal distributions.

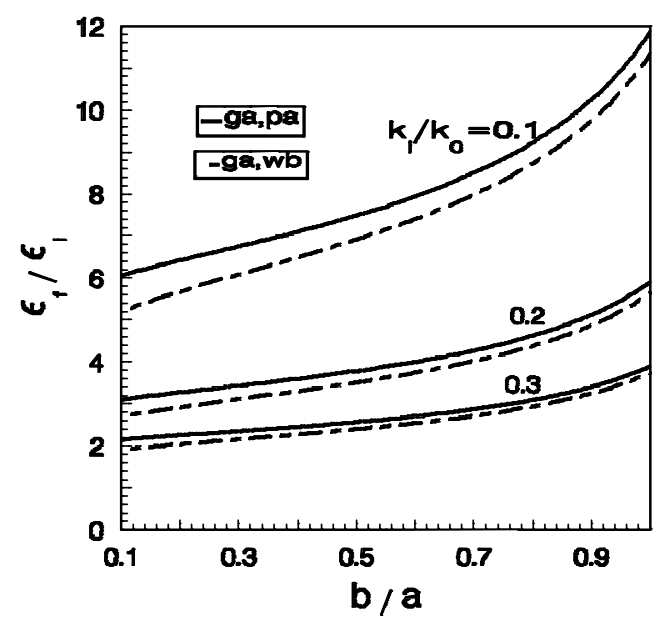

Figure 3: Emittance growth $\varepsilon_{\mathrm{f}} / \varepsilon_{\mathrm{i}}$ vs b/a for transverse GA distribution combining with either WB or PA longitudinal distribution.

\section{REFERENCES}

[1] Reiser M., Free energy and emittance growth in nonstationary charged particle beams, J. Appl. Phys., 1991, 70(4):1919-1923

[2] Chen Yinbao and Zhang Zhenhai, Emittance growth caused by the transverse nonuniform space charge distributions of bunched beam in linac, Chinese Journal of Nuclear Physics, 1994, 16(1):71-76

[3] Chen Yinbao, Fu Shinian, Huang Zhibin, et al., Space charge effect of bunched beam with nonuniform distributions in both longitudinal and transverse directions, Atomic Energy Science and Technology, 1994, 28(6):481-487. 